\title{
Actinidia eriantha Polysaccharide and PDI-Antibody Combination Therapy Enhances Antitumor Efficacy in Colorectal Cancer- Xenograft Mice
}

This article was published in the following Dove Press journal: OncoTargets and Therapy

Jinxia Li ${ }^{\prime}$

Yiping Wang ${ }^{2}$

Weiyang Jin ${ }^{3}$

Li Shen ${ }^{4}$

'College of Traditional Chinese Medicine, Hunan University of Chinese Medicine, Changsha, 4I0208, People's Republic of China; ${ }^{2}$ Key Laboratory of Digestive Pathophysiology of Zhejiang Province, First Affiliated Hospital of Zhejiang Chinese Medical University, Hangzhou, 310006, People's Republic of China; ${ }^{3} \mathrm{School}$ of Life and Environmental Science, Hangzhou Normal University, Hangzhou, 3III2I, People's Republic of China; ${ }^{4}$ Institute of Basic Theory of TCM, China Academy of Chinese Medical Sciences, Beijing, 100700, People's Republic of China
Correspondence: Li Shen

Institute of Basic Theory of TCM, China Academy of Chinese Medical Sciences, Beijing, 100700, People's Republic of China $\mathrm{Tel}+86-158-5825-6870$

Email shenlilII6@I26.com
Objective: To observe the efficacy of Actinidia eriantha polysaccharide (AEPS) combined with PD1 antibody therapy in colorectal cancer-xenograft mice.

Methods: CT26 cells were inoculated into $80 \mathrm{C} 57 \mathrm{BL} / 6$ mice to establish the colorectal cancer xenograft-mouse model. Mice were divided evenly into a model group, AEPS group, anti-PD1 group, and combined group. AEPS $5 \mathrm{~mL} / \mathrm{kg} \bullet$ day was given orally and $10 \mathrm{mg} / \mathrm{kg}$ anti-PD1 injected intravenously for 28 days. Tumor growth and mouse survival were observed. Tumor-cell proliferation and metastasis markers Ki67, N-cadherin, KLF4, and Oct4 were detected with immunochemistry and Western blotting, T-cell infiltration in spleens and tumors was detected with MTT and flow cytometry. IFN $\gamma$ and TNF $\alpha$ were detected with ELISA.

Results: Tumor growth was significantly retarded and survival prolonged in the AEPS, antiPD1, and combined groups. Ki67 expression decreased in the anti-PD1 and combined groups, and N-cadherin, KLF4, and Oct4 expression decreased in the AEPS and combined groups. IFN $\gamma$ and TNF $\alpha$ levels, T-cell infiltration in spleen, and tumor all increased distinctively in the AEPS and combined groups. The combined group showed better antitumor effects and life-extension effect than the other two groups.

Conclusion: AEPS and PD1 antibody-combination therapy can suppresses tumor growth and prolong survival of colorectal cancer-xenograft mice by regulating immunofunction, and the combined therapy showed better therapeutic efficacy than the single treatment.

Keywords: colorectal cancer, PD1 antibody, Actinidia eriantha polysaccharide, combined therapy

\section{Introduction}

Colorectal cancer is a common malignant tumor in the digestive system. With improvements in living standards and changes in dietary habits, the incidence of colorectal cancer has been increasing year by year. Chinese cancer statistics in 2018 showed that colorectal cancer is the third-most malignant tumor in China. ${ }^{1}$ Radical resection is the first choice for colorectal cancer, but recurrence and 5-year survival are improving. Moreover, tumors are usually found in the advanced or late stage, when excision is no longer appropriate. ${ }^{2}$ Tumor immunotherapy, especially the application of immunocheckpoint inhibitors, has become a focus in tumor research. High expression of PD1 can inhibit lymphocyte infiltration, which is an important 
mechanism in tumor immunoescape. The PD1 antibody has been clinically applied in the treatment of multiple kinds of tumor, due to its advantages of strong specificity, small side effects, and long duration of action. ${ }^{3-6}$ However the effectiveness of PD1 in different cancer species or different patients is quite different, and some patients even have pseudotumor progression. ${ }^{7}$

Reasonably combined immunotherapy may be a solution to improve clinical antitumor efficacy. Hypoimmunity is closely related to the pathological evolution of colorectal cancer. TheT cell-conversion rate and circulating immunocomplex in colorectal cancer patients are significantly lower than in healthy people, ${ }^{8}$ suggesting that PD1 combined with an immunoenhancing drug is a promising prospect for the treatment of colorectal cancer. The root of Actinidia eriantha is a kind of traditional Chinese medicine (TCM) and has been widely used in treating various malignant tumors. A. eriantha polysaccharide (AEPS) is the active component of $A$. eriantha and has been reported to have antitumor effects. AEPS inhibits the Wnt-signaling pathway and reduces the proliferation and induces cell apoptosis of colon cancer cells. ${ }^{9}$ AEPS improves immunofunction by regulating NFKB and IL12 $\beta$, downregulates the expression of PCNA, P53, and BCL2, and inhibits proliferation while inducing apoptosis in gastric cancer mice. ${ }^{10,11}$ Therefore, PD1 combined with AEPS could be useful in treating colorectal cancer.

In this study, PD1 combined with AEPS therapy was introduced in colorectal cancer-xenograft mice and effectiveness and mechanisms investigated. We found that AEPS and PD1 combination therapy suppressed tumor growth and prolonged survival of model mice by regulating their immunofunction. The combined therapy showed better therapeutic efficacy than the single treatment. AEPS combined with PD1 therapy could be feasible in the treatment of colorectal cancer.

\section{Methods}

\section{Reagents}

AEPS was provided by the laboratory of Professor Zhaohuan Lou, Zhejiang University of Traditional Chinese Medicine, anti-PD1 nivolumab was purchased from Selleck Chemicals (Houston, TX, USA), anti- $\beta$ actin, anti-Oct4, anti-KLF4, and anti-N-cadherin from Cell Signaling Technology (Danvers, MA, USA), mouse IFN $\alpha$ and TNF $\alpha$ ELISA kit from eBioscience (San Diego, CA, USA), and anti-Ki67 antibody and anti-CD8 $\alpha$ antibody from Abcam (Cambridge, UK).

\section{Animals}

Eighteen male C57BL/6 mice weighing 18-22 g were purchased from and fed in the animal experiment center of Zhejiang University of Traditional Chinese Medicine. Animals were housed in a specific pathogen-free environment and fed normal water and food. All procedures received the approval of the Laboratory Animal Management and Welfare Ethical Review Committee of Zhejiang University of Traditional Chinese Medicine (ZJCLA-IACUC-20010013).

\section{Mouse Model and Grouping}

CT26 cells were purchased from the type culture collection of the Chinese Academy of Sciences (Shanghai, China). Cells at logarithmic growth stage were prepared into single-cell suspensions, cell numbers was counted using flow cytometry, and the percentage of living cells was $>95 \%$. Cell density was adjusted to $2 \times 10^{7} / \mathrm{mL}$ and inoculated into subcutaneous tissue of each mouse at $0.1 \mathrm{~mL}$ per mouse. Tumor growth to about $100 \mathrm{~mm}^{3}$ was considered successful modeling.

Mice were randomly divided into a model group, AEPS group, anti-PD1 group, and combined group based on a random-number table, with 20 mice in each group. Mice in the AEPS group were given $5 \mathrm{~mL} / \mathrm{kg}$ AEPS orally per day for 28 days, mice in anti-PD1 group were given a $10 \mathrm{mg} / \mathrm{kg}$ caudal intravenous injection once a week for 4 weeks, mice in the combined group were given combination therapy of AEPS and PD1 at the same dosages, and mice in the model group were injected with the same volume of normal saline through the tail vein. On the 28th day, ten mice from each group were killed for subsequent testing. The remaining mice continued to receive the drug until natural death.

\section{Body Weight and Spleen Index}

Mice were weighed every 3 days. On the 28th day, mice were anesthetized with pentobarbital sodium, blood taken from the aorta abdominalis, and spleens and tumors quickly removed under aseptic conditions. Finally, mice were killed by carbon dioxide euthanasia. Spleens were washed and weighed, and the spleen index calculated: spleen weight/body weight $\times 100 \%$.

\section{Tumor Growth and Survival Rate}

The length (a) and width (b) of tumors were measured twice a week with Vernier calipers. The tumor volume was 
calculated according to the formula $\mathrm{V}=\mathrm{a} \times \mathrm{b}^{2} \times 0.5$, and the tumor-growth curve was drawn. On the 28th day, tumor tissue was removed and weighed, and tumorinhibition rate calculated: (tumor weight of control group - tumor weight of experimental group)/tumor weight of control group $\times 100 \%$. The remaining mice were given drugs continuously until natural death and survival recorded.

\section{MTT Assay of Proliferation of T Lymphocytes in Mouse Spleen}

Spleens were removed in sterile conditions and placed in a small dish containing sterile Hank's solution. Tweezers was used to gently shred the spleen into a single-cell suspension, which was then filtered through a 200-mesh sieve, washed, and the cell concentration adjusted to $3 \times 10^{6} / \mathrm{mL}$ with RPMI 1640 culture medium. Then, $1 \mathrm{~mL}$ cell suspension was added to two wells each and 7$5 \mathrm{~mL}$ ConA solution $(7.5 \mathrm{~g} / \mathrm{mL})$ added to one well. The other well was used as a control. The cell suspension was cultured for 68 hours in an incubator at $37^{\circ} \mathrm{C}$ and $5 \% \mathrm{CO}_{2}$. Then, $0.7 \mathrm{~mL}$ supernatant was gently sucked from each well, culture medium without calf serum and $5 \mathrm{mg} / \mathrm{mL}$ MTT added, and culturing continued for another 4 hours. After this, $1 \mathrm{~mL}$ acidic isopropanol was added to each well and blown evenly to completely dissolve the purple crystals. Optical density (OD) was measured at $570 \mathrm{~nm}$ wavelength. Finally, the proliferation ability of $\mathrm{T}$ lymphocytes was calculated by subtracting the OD values with or without ConA holes.

\section{ELISA of IFN $\gamma$ and TNF $\alpha$}

Standard and serum samples $(100 \mu \mathrm{L})$ were added to a 96well plate, $50 \mu \mathrm{L}$ each enzyme added to each well for 1 hour's incubation, the liquid removed, the plate washed times, $50 \mu \mathrm{L}$ substrate $\mathrm{A}$ and $\mathrm{B}$ added and incubated at $36^{\circ}$ $\mathrm{C} \pm 2^{\circ} \mathrm{C}$ in the dark for $15 \mathrm{~min}$, and $50 \mu \mathrm{L}$ termination solution added. The OD value was measured at a wavelength of $450 \mathrm{~nm}$.

\section{Immunohistochemical Analysis of Ki67}

Tumor sections were routinely dewaxed and hydrated. After heat-mediated antigen retrieval, sections were washed with PBS three times and blocked with 5\% BSA at $37^{\circ} \mathrm{C}$ for 30 minutes.,Sections were incubated with $\mathrm{Ki} 67$ antibody $(1: 1,000)$ at $4^{\circ} \mathrm{C}$ overnight, then washed with PBS 3 times and SABC incubated with streptavidin-biotin at $37^{\circ} \mathrm{C}$ for 45 minutes, followed by diaminobenzidine coloration and hematoxylin restaining. Integral OD was analyzed with Image-Pro Plus 6.0.

\section{Immunofluorescence Staining of $\mathrm{CD}^{+}$ $\mathrm{T}$ Lymphocytes in Tumor Tissue}

Antigen retrieval and blockage were performed as before. Sections were incubated with anti-CD8 $\alpha$ antibody at 1:800 dilution and $4{ }^{\circ} \mathrm{C}$ overnight, followed with fluorescencelabeled secondary antibody incubation. Slices were observed and images collected by confocal microscopy.

\section{Western Blotting Analysis of N-Cadherin, KLF4, and Oct4}

Tumor tissue was homogenized and total protein extracted. Concentration of proteins was determined with bicinchoninic acid assay. Proteins were separated by SDS-PAGE and transferred to polyvinylidene fluoride membrane, then $5 \%$ BSA was used for blocking, membranes incubated with N-cadherin (1:1,000), KLF4 (1:1,000), Oct4 (1:800), and $\beta$-actin $(1: 3,000)$ primary antibodies at $4^{\circ} \mathrm{C}$ overnight. Then membranes were washed and incubated with secondary antibodies $(1: 1,000)$ for 2 hours. Integrated OD was detected with a far-infrared scanner (ChemiDoc Touch Imaging System, Bio-Rad) and ImageJ. Relative protein expression was calculated using $\beta$-actin as internal control.

\section{Statistical Analysis}

Statistical analysis was performed using SPSS 20.0. Data are presented as means $\pm \mathrm{SD}$. One-way ANOVA was used for comparison of tumor volume measured at different times between the groups, and $t$-tests for comparison of data between groups. $P<0.05$ was considered statistically significant. Log-rank analysis was used to evaluate the relationship between different intervention and prognosis, and Kaplan-Meier analysis was used to analyze the survival curve.

\section{Results}

\section{Combination of AEPS and PDI Inhibited} Tumor Growth in Mice

Tumor volume, weight, and inhibition rate were observed to comprehensively evaluate the effectiveness of AEPS and PD1 combined therapy on tumor growth. Shapes of tumors are shown in Figure 1A. To dynamically measure the growth pattern of tumors, as shown in Figure 1B and $\mathrm{C}$, tumors in the three treatment groups 
grew more slowly than the control group. Tumor volume in the control, AEPS group, anti-PD1, and combined groups on the 28th day was $877.93 \pm 114.95 \mathrm{~mm}^{3}$, $732.64 \pm 72.16 \mathrm{~mm}^{3}, 518.84 \pm 171.56 \mathrm{~mm}^{3}$, and 309.20 $\pm 74.02 \mathrm{~mm}^{3}$, respectively. Tumor weight in the four groups was $0.80 \pm 0.10 \mathrm{~g}, 0.67 \pm 0.13 \mathrm{~g}, 0.39 \pm 0.08 \mathrm{~g}$, and $0.26 \pm 0.09 \mathrm{~g}$, respectively. This indicated that tumors in the anti-PD1 and combined groups were smaller and lighter than the control group $(P<0.01)$, and values in the combined group were lower than the anti-PD1 group $(P<0.05)$. As shown in Figure 1D, the tumor-inhibition rate in the combined group was significantly higher than the AEPS and anti-PD1 groups $(P<0.05)$. These results suggest that AEPS with PD1 combination therapy
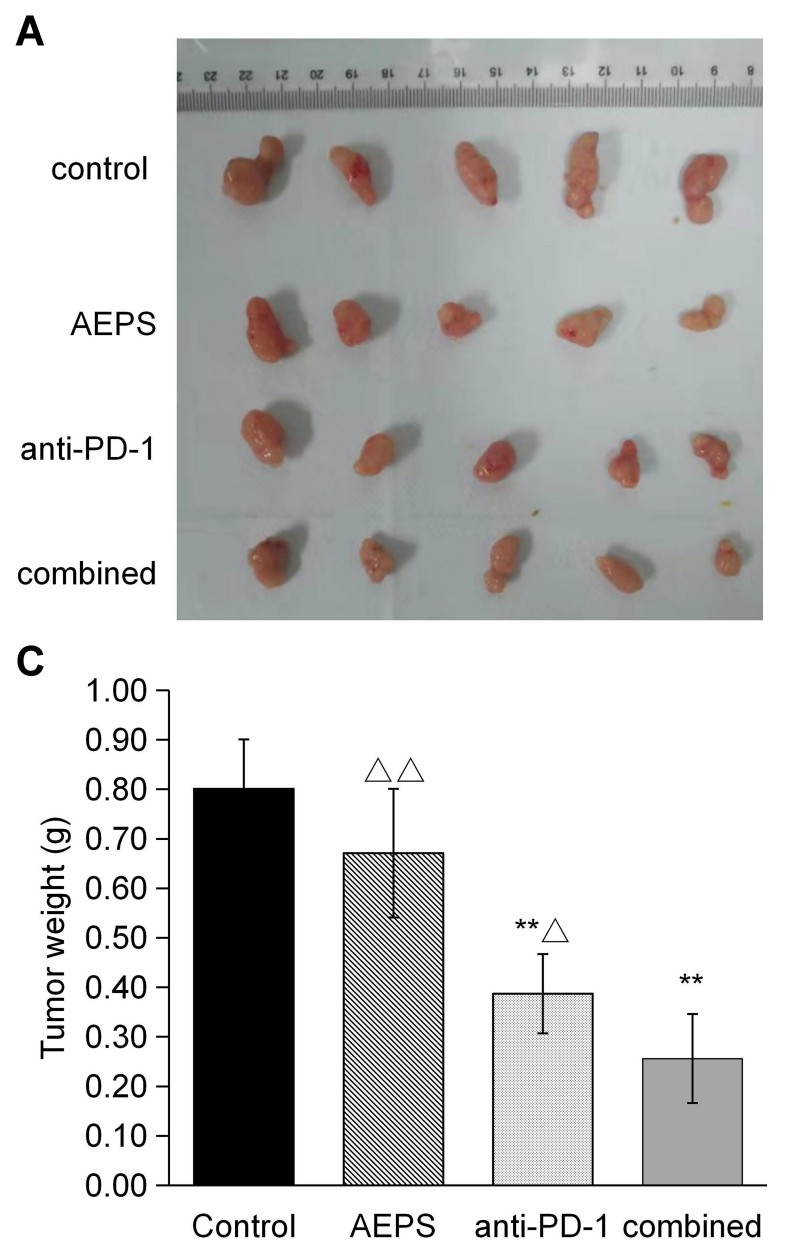

effectively suppressed tumor growth in colorectal cancer-xenograft mice, and showed better therapeutic efficacy than single treatment with PD1 or AEPS.

\section{Combination of AEPS and PDI Prolonged Survival in Mice}

As shown in Figure 2, average survival in the control, AEPS, anti-PD1, and combined groups was 74.8 \pm 7.7 days, $89.8 \pm 8.9$ days, $108.0 \pm 12.3$ days, and 121.4 \pm 12.6 days, respectively. Survival in all three treatment groups was significantly longer than the control group $(P<0.05)$ and survival in the combined group was longer than the AEPS group $(P<0.01)$.

B
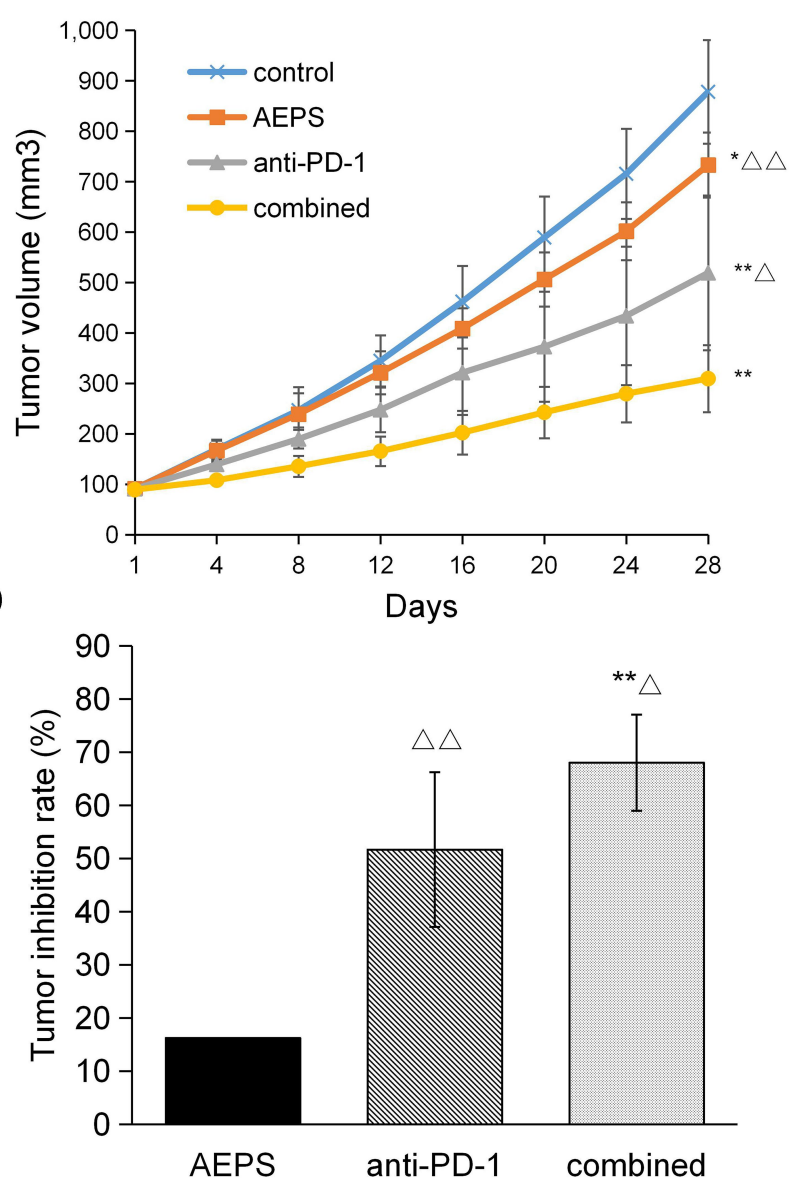

Figure I Combination of AEPS and PDI antibody inhibited tumor growth in colorectal cancer-xenograft mice. CT26 cells were inoculated to establish the colorectal the cancer-xenograft mouse model, $5 \mathrm{~mL} / \mathrm{kg}$-day AEPS was given orally orally, and $10 \mathrm{mg} / \mathrm{kg} \cdot$ week anti-PDI injected intravenously intravenously separately or combined for 28 days. (A) After 28 days, mice were killed and tumors arranged and photographed. (B) Tumor volumes were measured at the indicated times using Vernier calipers: volume $=$ length $\times$ width $^{2} / 2$. (C) Average tumor weights were analyzed in each group. (D) Tumor-inhibition rates were calculated in each group: inhibition rate $=$ (tumor weight of control group - tumor weight of experimental group)/tumor weight of control group $\times 100 \%$. Statistical analysis was performed using Student's $t$-test and one-way ANOVA. $* P<0.05, * * P<0.01$ compared to the control group; ${ }^{\Delta} P<0.05,{ }^{\Delta \Delta} P<0.01$ compared to the combined group. 


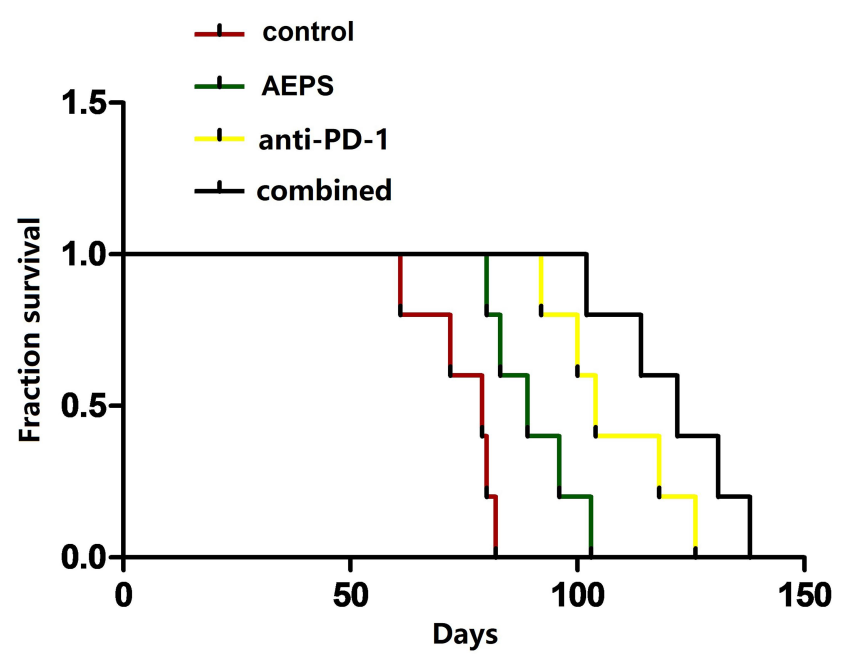

Figure 2 Combination of AEPS and PDI antibody prolonged survival in colorectal cancer-xenograft mice. CT26 cells were inoculated to establish the colorectal cancer-xenograft mouse model, $5 \mathrm{~mL} / \mathrm{kg} \cdot$ day AEPS was given orally and $10 \mathrm{mg} /$ $\mathrm{kg}$ *week anti-PDI injected intravenously separately or combined until the mouse was dead. Their survival was recorded individually.

\section{Combination of AEPS and PDI Postponed Body-Weight Gain and Increased Spleen Index in Mice}

Weight of mice were measured dynamically every 3 days, as shown in Figure 3A. Weight gain in mice in the three treatment groups was slightly slower than the control group, but not statistically significant. Mental and physiological conditions of the mice receiving treatment were better than the controls. As for spleens, as shown in Figure $3 \mathrm{~B}$, compared to the control group, spleens in the AEPS,
anti-PD1, and combined groups were significantly enlarged $(P<0.05)$, and values in the combined group were highest $(P<0.05)$.

\section{Combination of AEPS and PDI Inhibited Tumor-Proliferation and \\ Metastasis-Marker Protein Expression}

Immunohistochemistry was used to determine the expression of the tumor-proliferation marker Ki67. Western blotting was used to detect the expression of the tumor-metastasis markers N-cadherin, KLF4, and Oct4. As shown in Figure 4, compared with the control group, Ki67 expression in the anti-PD1 group and combined group andN-cadherin, KLF4 and Oct4 expression in the AEPS and combined groups were all significantly decreased $(P<0.05)$, and the combined group had the lowest values $(P<0.05)$. These results suggest that the combined therapy reduced the expression of tumorproliferation and metastasis-marker proteins, and so inhibited colorectal tumor growth.

\section{Combination of AEPS and PDI Stimulated Spleen T-Lymphocyte Proliferation and Tumor $\mathrm{CD}^{+}$}

\section{T-Lymphocyte Infiltration}

ConA was used to induce the proliferation of spleen lymphocytes, which was detected by MTT. As shown in Figure $5 \mathrm{~A}$, compared with the control group, the three treatment groups all showed significantly increased proliferation of

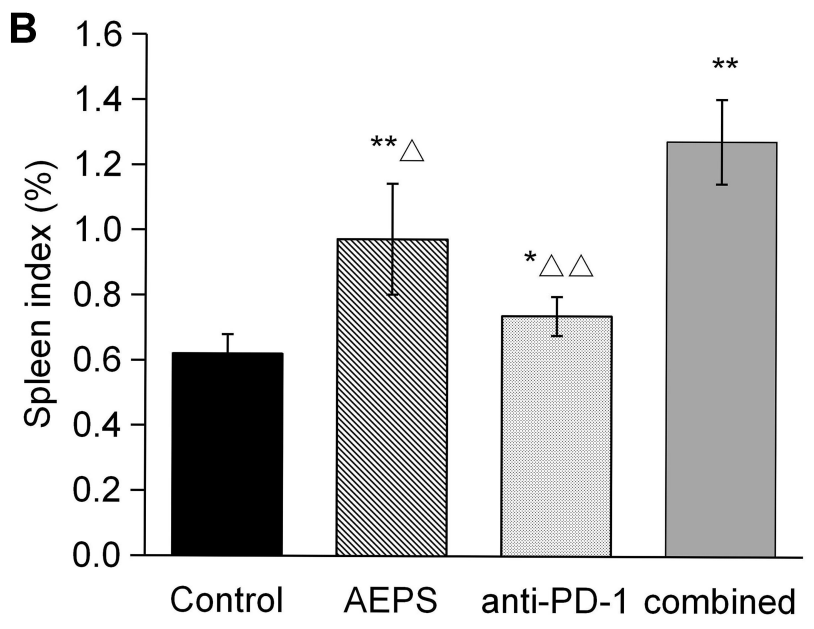

Figure 3 Combination of AEPS and PDI antibody postponed body-weight gain and increased spleen index in colorectal cancer-xenograft mice. CT26 cells were inoculated to establish the colorectal cancer-xenograft mouse model, $5 \mathrm{~mL} / \mathrm{kg} \cdot$ day AEPS was given orally, and $10 \mathrm{mg} / \mathrm{kg} \cdot$ week anti-PDI injected intravenously separately or combined for 28 days. (A) Body weights were measured at the indicated times. (B) After 28 days, mice were killed and spleens removed and weighed. Statistical analysis was performed using Student's t-test. $* P<0.05, * * P<0.01$ compared to the control group; ${ }^{\Delta} P<0.05,{ }^{\Delta \Delta} P<0.01$ compared to the combined group. 
A
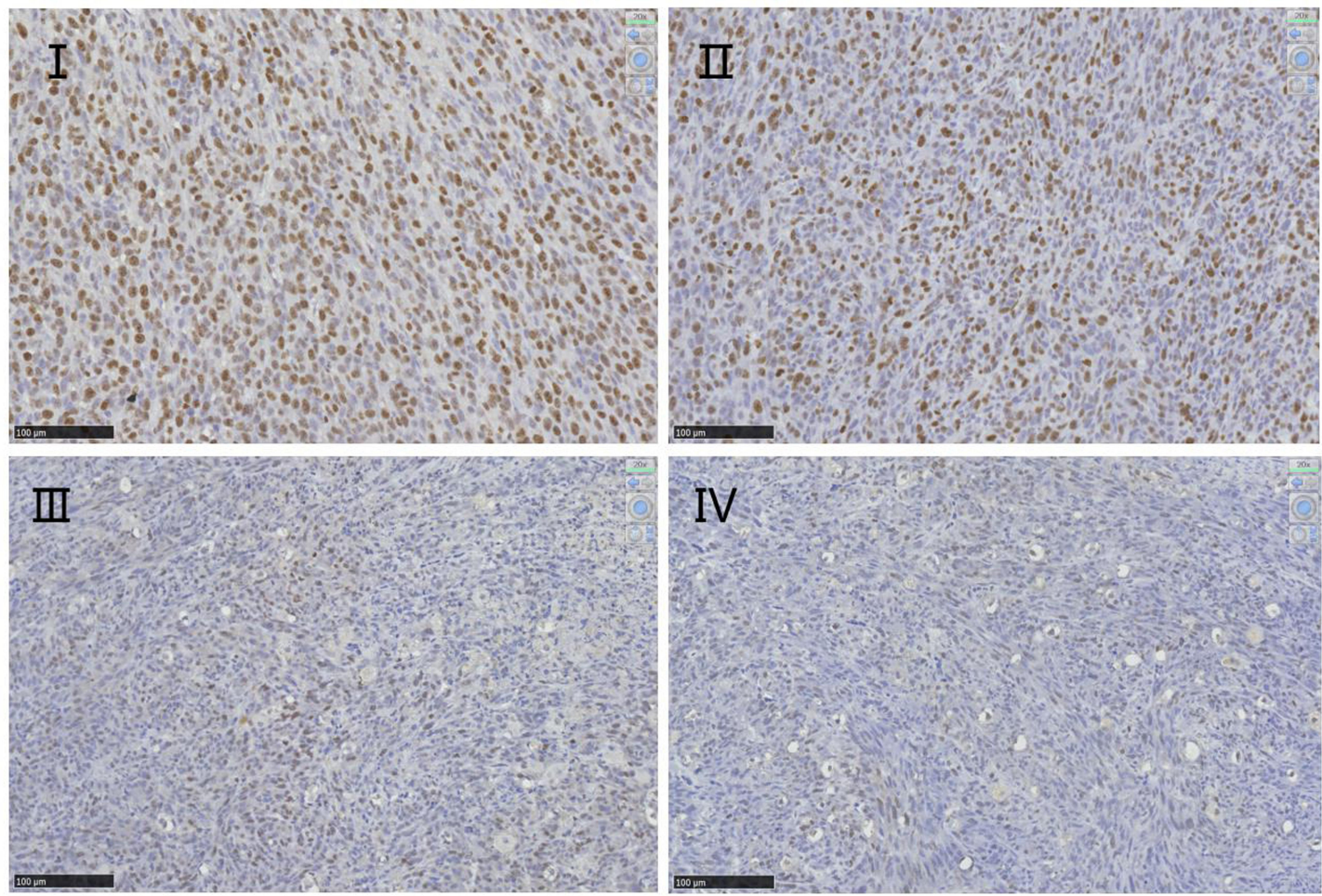

B

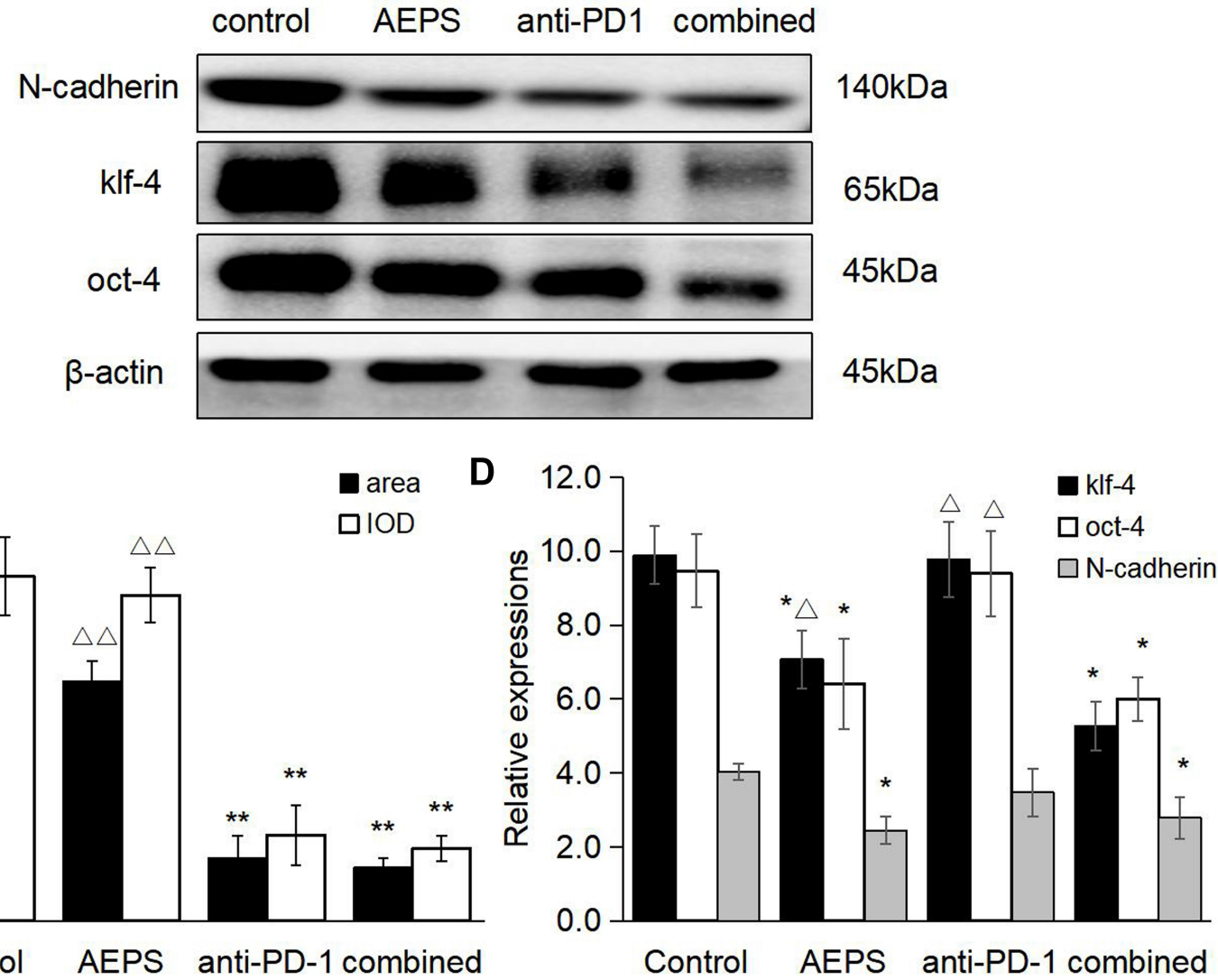

Figure 4 Combination of AEPS and PDI antibody inhibited tumor proliferation and metastasis-marker protein expression in colorectal cancer-xenograft mice. CT26 cells were inoculated to establish the colorectal cancer-xenograft mouse model, $5 \mathrm{~mL} / \mathrm{kg} \cdot$ day AEPS given orally, and $10 \mathrm{mg} / \mathrm{kg} \cdot$ week anti-PDI injected intravenously separately or combined for 28 days. (A) After 28 days, tumors in each group were removed and sliced, IHC staining performed with the anti-Ki67 primary antibody, and slices observed and photographed under $200 \times$ magnification. (I) control group, (II) AEPS group, (III) anti-PDI group, (IV) combined group. (B) Western blot was performed with anti-N-cadherin, anti-KLF4, and anti-Oct4 primary antibodies. (C) Area and integral optical density of Ki67 was analyzed with Image-Pro Plus 6.0. (D) Semiquantification of protein levels was performed with Imagej software, and relative expressions of proteins calculated using $\beta$-actin as an internal control. Statistical analysis was performed using Student's $t$-test. $* P<0.05$, $* * P<0.0$ I compared to the control group; ${ }^{\Delta} P<0.05$, ${ }^{\triangle \triangle} P<0.01$ compared to the combined group. 
A

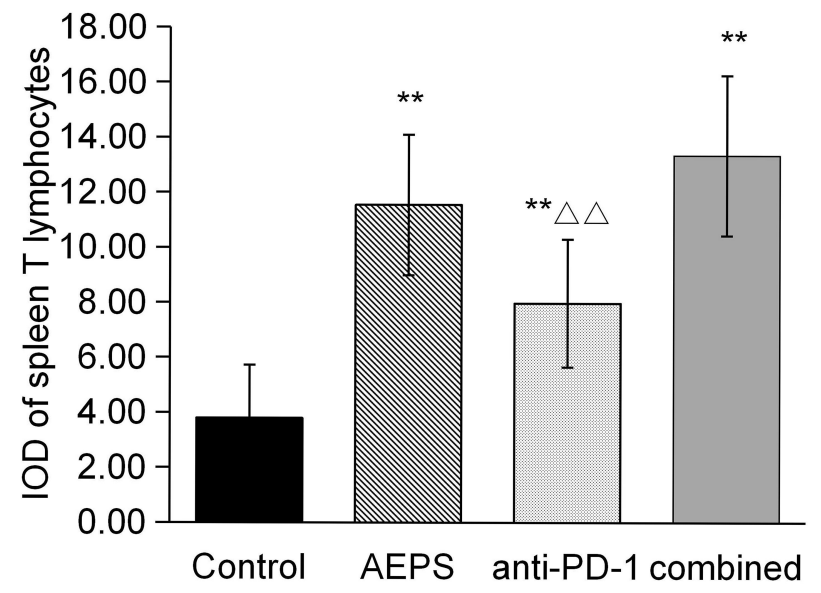

B

CD8
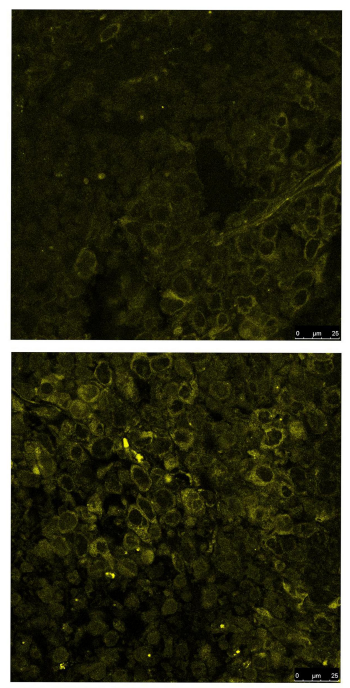

AEPS

anti-PD-1
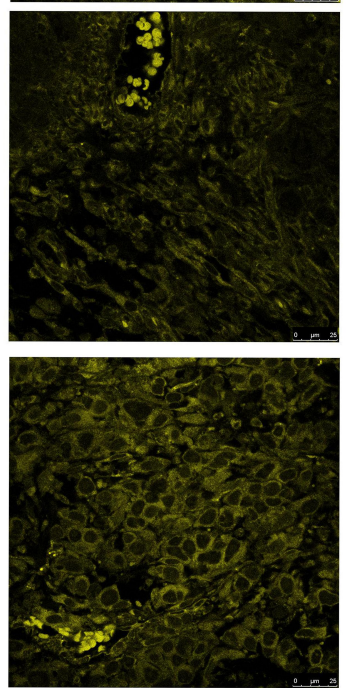

DAPI
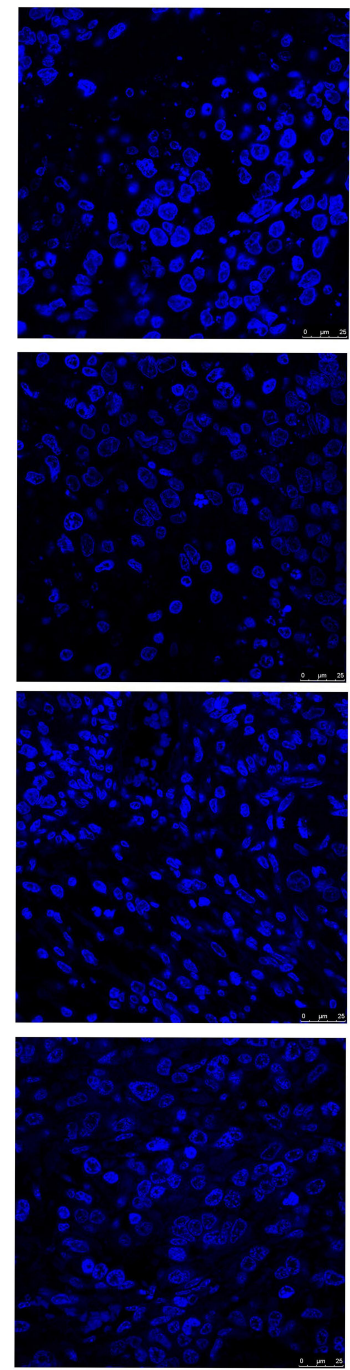

Merge
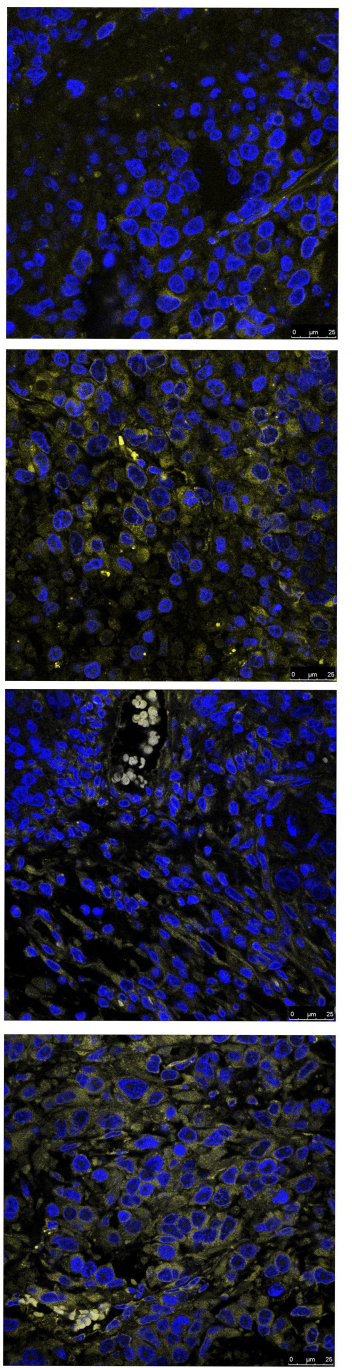

Figure 5 Combination of AEPS and PDI antibody stimulated spleen T-cell proliferation and tumor CD8 ${ }^{+}$T-cell infiltration in colorectal cancer-xenograft mice. CT26 cells were inoculated to establish the colorectal cancer-xenograft mouse model, $5 \mathrm{~mL} / \mathrm{kg} \cdot$ day AEPS was given orally, and $10 \mathrm{mg} / \mathrm{kg} \cdot$ week anti-PDI injected intravenously separately or combined for 28 days. (A) After 28 days, spleens were removed and cells extracted and cultured. ConA was used to induce the proliferation of spleen lymphocytes, proliferation activity of T cells was detected with MTT, and optical density measured at $570 \mathrm{~nm}$ wavelength. Statistical analysis was performed using Student's $t$-test. $* * P<0.0$ l compared to the control group; ${ }^{\Delta \Delta} P<0.0$ I compared to the combined group. (B) Immunofluorescence analysis was performed with anti-CD8 $\alpha$ primary antibody, coverslipped slides were covered with DAPI, and merged images show the overlap of yellow CD8 and blue DAPI fluorescence. 
spleen T lymphocytes $(P<0.05)$. Transplanted tumors were sliced and immunofluorescence staining performed to detect tumor-infiltrated $\mathrm{CD}^{+} \mathrm{T}$ cells. As shown in Figure 5B, tumor-infiltrated $\mathrm{CD}^{+} \mathrm{T}$ cells in the AEPS and combined groups were significantly increased compared with the control group. These results suggest that the combination of AEPS and PD1 increased the number of $\mathrm{T}$ lymphocytes in spleens and tumors and activated immunity in colorectal cancer-xenograft mice.

\section{Combination of AEPS and PDI Increased Levels of Immunoregulatory Cytokines in Mice}

$\mathrm{TNF} \alpha$ and IFN $\gamma$ content was measured with ELISA. As shown in Figure 6, compared with the control group, anti-
PD1 increased IFN $\gamma$, AEPS increased TNF $\alpha$, and combined therapy raised both $\mathrm{TNF} \alpha$ and $\operatorname{IFN} \gamma(P<0.01)$, indicating that the combination of AEPS and PD1 promoted the release of immunoregulatory cytokines and activated the immunoresponse in colorectal cancer-xenograft mice.

\section{Discussion}

Immunocheckpoint blockade is an advanced strategy in cancer management. Recent advances have indicated that the expression of immunoinhibitory checkpoints, such as PD1/PDL1 function as potent mediators for the balance and escape phases of cancer immunoediting. Antibodies targeting the PDL1-PD1 axis have been evaluated in $>1,000$ clinical trials and have been approved for several cancer treatments. ${ }^{12}$ Polysaccharides from TCM have been widely reported to inhibit the proliferation and metastasis
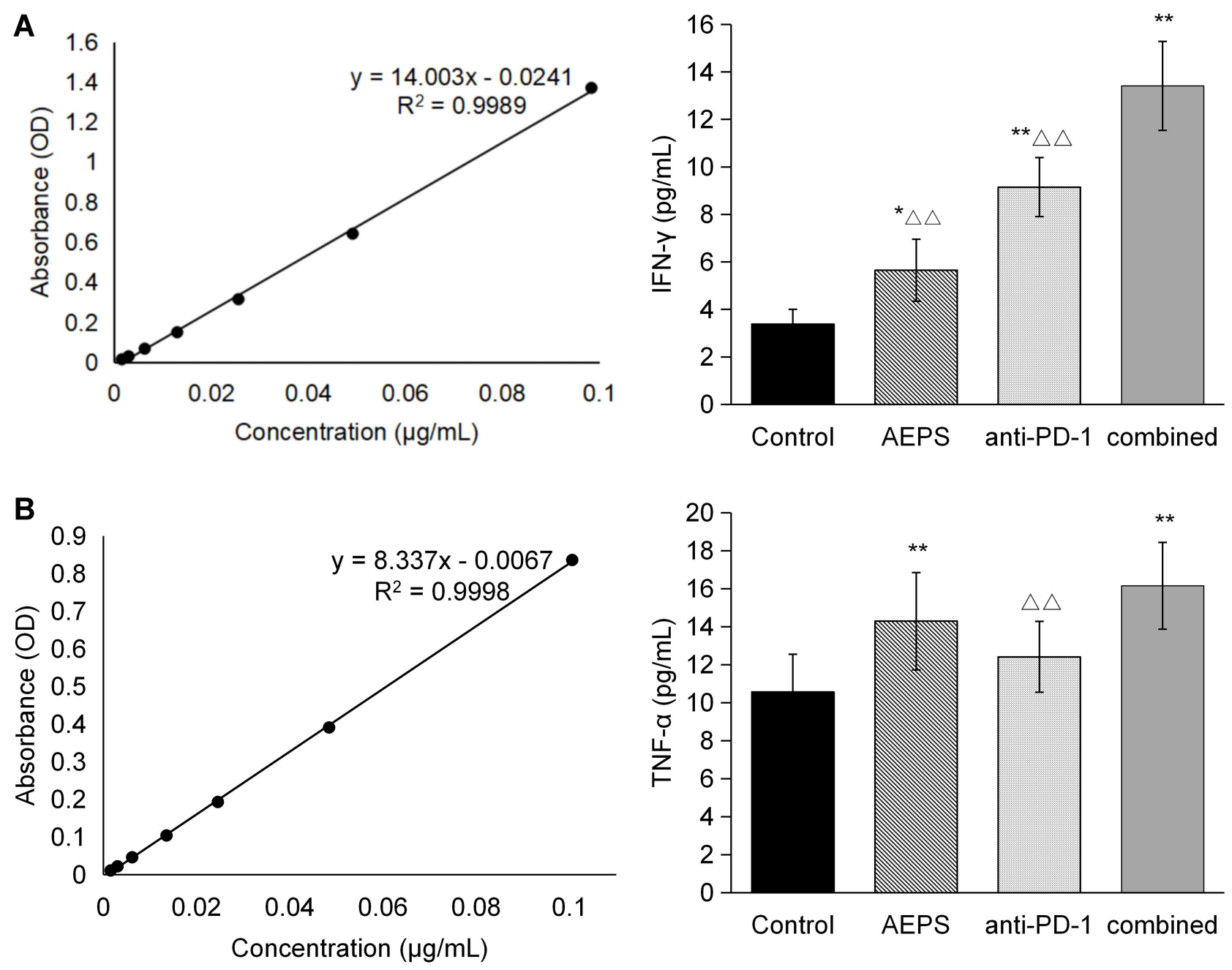

Figure 6 Combination of AEPS and PDI antibody increased serum levels of TNF $\alpha$ and IFN $\gamma$ in colorectal cancer-xenograft mice. CT26 cells were inoculated to establish colorectal the cancer-xenograft mouse model, $5 \mathrm{~mL} / \mathrm{kg} \bullet$ day AEPS was given orally, and $10 \mathrm{mg} / \mathrm{kg} \bullet$ week anti-PDI injected intravenously separately or combined for 28 days. After 28 days, blood was collected and serum isolated. (A) IFN $\gamma$ and (B) TNF $\alpha$ were analyzed with ELISA. Statistical analysis was performed using Student's $t$-test. $* P<0.05$, $* * P<0.01$ compared to the control group; ${ }^{\Delta \Delta} \mathrm{P}<0.01$ compared to the combined group. 
of cancer cells and restore immunofunction. ${ }^{13}$ Moreover, with low toxicity and small side effects, TCM can increase therapeutic efficacy while reducing the toxicity of chemotherapy drugs, which makes it a good adjuvant anticancer strategy. ${ }^{14}$ The root of A. eriantha is a kind of TCM herb that is frequently used clinically in the treatment of gastrointestinal tumors. AEPS is extracted from the roots of $A$. eriantha whose aldoses or ketoses are linked by glycoside bonds, endowing it with a variety of biological activities. AEPS has been reported to be a potential antitumor agent with immunomodulatory activity; ${ }^{15,16}$ therefore, AEPS combined with PD1 may be an effective anticancer therapy in the treatment of colorectal cancer. This study used the CT26 cell line to establish a xenograft mouse model of colorectal cancer. AEPS + PD1 combined therapy was applied. The results showed that the combined therapy remarkably decreased the volume and weight of transplanted tumors and significantly prolonged the survival of mice. Antitumor effects and life extension were better than with single use of AEPS or PD1. The outcomes verified the effectiveness of AEPS and PD1 combination therapy in colorectal cancerxenograft mice, and could be useful for the further treatment of colorectal cancer in clinical practice.

The antitumor effects of AEPS and PD1 combination therapy were achieved by suppressing expression of tumor-proliferation and metastasis-marker proteins. The combined therapy significantly inhibited the expression of proliferation marker Ki67 and metastasis markers N-cadherin, KLF4, and Oct4, and values were further decreased compared with the anti-PD1 and AEPS groups.

The antitumor mechanism of AEPS and PD1 combination therapy is related to the regulation of immunofunction. Under normal circumstances, the immune system in lymph nodes or spleen responds to foreign antigens and promotes the proliferation of antigen-specific $\mathrm{T}$ cells. Activation of PD1 conducts inhibitory signals and reduces the proliferation of $\mathrm{T}$ cells, allowing cancer cells to evaded immunosurveillance and destruction. On the contrary, blockage of the PD1PDL1 signal pathway may reverse the tumor immunomicroenvironment and enhance endogenous antitumor immunoeffects, eventually inhibiting tumor progression. ${ }^{17,18}$ AEPS has also been reported toregulate immunocytokines and enhance immunofunction. ${ }^{16}$ In this study, we introduced AEPS and PD1 combined therapy. In the combined group, spleens significantly enlarged, spleen-lymphocyte proliferation and tumor-infiltrated $\mathrm{CD} 8^{+} \mathrm{T}$ cells distinctly increased, and immunoregulating cytokines TNF $\alpha$ and IFN $\gamma$ significantly increased, indicating that AEPS and PD1 combination therapy can enhance the immunofunction of colorectal cancer-xenograft mice by regulating proliferation and metastasis of tumor cells and suppressing the growth of tumor tissue.

\section{Conclusion}

AEPS combined with PD1 can suppresses tumor growth and extend survival of colorectal cancer-xenograft mice by regulating their immunofunction, and this therapy showed better therapeutic efficacy in treating colorectal cancer than single-agent treatments.

\section{Abbreviations}

AEPS, A. eriantha polysaccharide; TCM, traditional Chinese medicine; OD, optical density.

\section{Data Sharing Statement}

The materials and data of this study are available to other researchers upon request to Li Shen, shenli1116@126.com.

\section{Ethics Approval and Consent to Participate}

This study was approved (ZJCLA-IACUC-20010013) by the Laboratory Animal Management and Welfare Ethical Review Committee of Zhejiang University of Traditional Chinese Medicine. For an animal carrying one tumor, the diameter should not exceed $2 \mathrm{~cm}$ in mice for therapeutic studies according to the guidelines from the University of Pennsylvania Institutional Animal Care and Use Committee. This study was carried out in accordance with the local guidelines of the Welfare Ethical Review Committee of Zhejiang University of Traditional Chinese Medicine.

\section{Consent for Publication}

All authors have provided consent for publication.

\section{Author Contributions}

All authors made substantial contributions to conception and design, acquisition of data, or analysis and interpretation of data, took part in drafting the article or revising it critically for important intellectual content, agreed to submit to the current journal, gave final approval to the version to be published, and agree to be accountable for all aspects of the work. 


\section{Funding}

This study was supported by the National Natural Science Foundation of China (82074398, 81873276, 81703753) and the Natural Science Foundation of Zhejiang Province (LY20H280006).

\section{Disclosure}

The authors have no conflicts of interest related to this work to report.

\section{References}

1. Feng RM, Zong YN, Cao SM, et al. Current cancer situation in China: good or bad news from the 2018 global cancer statistics? Cancer Commun (Lond). 2019;39(1):22. doi:10.1186/s40880-019-0368-6

2. Haraldsdottir S, Einarsdottir HM, Smaradottir A, et al. Colorectal cancer - review. Laeknabladid. 2014;100(2):75-82. doi:10.17992/ lbl.2014.02.531

3. Arlauckas SP, Garris CS, Kohle RH, et al. In vivo imaging reveals a tumor-associated macrophage-mediated resistance pathway in anti-PD-1 therapy. Sci Transl Med. 2017;9(389):3604. doi:10.1126/ scitranslmed.aal3604

4. Ding J, Jiang JT. The antitumor immunological mechanism of programmed death 1/programmed death ligand 1 monotherapy combined with radiotherapy. Chin J Exp Surg. 2018;35(12):2362.

5. Nanda R, Chow LQ, Dees EC, et al. Pembrolizumab in patients with advanced triple-negative breast cancer: phase Ib KEYNOTE-012 study. J Clin Oncol. 2016;34(21):2460. doi:10.1200/ JCO.2015.64.8931

6. Tahara M, Muro K, Hasegawa Y, et al. Pembrolizumab in Asia-Pacific patients with advanced head and neck squamous cell carcinoma: analyses from KEYNOTE- 01. Cancer Sci. 2018;109(3):771. doi: $10.1111 /$ cas. 13480

7. Millrud CR, Bergenfelz C, Leandersson K. On the origin of myeloid-derived suppressor cells. Oncotarget. 2017;8(2):3649. doi:10.18632/oncotarget.12278
8. Koi M, Carethers JM. The colorectal cancer immune microenvironment and approach to immunotherapies. Future Oncol. 2017;13 (18):1633-1647. doi:10.2217/fon-2017-0145

9. Xu L, Lidan L, Xianhui S. Actinidia Chinensis Polysaccharide regulates Wnt signaling pathway to inhibit proliferation and promote apoptosis of colon cancer. Chin J Gerontol. 2019;39(9):2215-2218.

10. Guangji Z, Li S, Guangshun Z, et al. Influence of actinidia chinensis polysaccharide on expression of PCNA and p53 in orthotopic transplanted cancer of gastric tumor in 615 mice. Chin J Tradit Chin Med Pharm. 2013;28(9):2538-2541.

11. Li S, Guangji Z, Guangshun Z, et al. Effect of Actinidia chinensis polysaccharide on apoptosis of MFC and their orthotopic transplanted tumor of gastric cancer. Chin Tradit Herbal Drugs. 2014;45 (5):673-678.

12. Alsaab HO, Sau S, Alzhrani R, et al. PD-1 and PD-L1 checkpoint signaling inhibition for cancer immunotherapy: mechanism, combinations, and clinical outcome. Front Pharmacol. 2017;8:561. doi:10.3389/fphar.2017.00561

13. Haoyue L, Jinxia L, Li S. Effect of Chinese medicine polysaccharides on tumor microenvironment. J Zhejiang Chin Med Univ. 2019;43 (9):1010-1014.

14. Zhao H. Brief analysis on the research significance of Actinidia Chinensis Polysaccharide in the treatment of gastric carcinoma. Guang Min J Chin Med. 2017;32(13):1833-1834.

15. Du J, Chen X, Ye Y, et al. A comparative study on the mechanisms of innate immune responses in mice induced by alum and actinidia eriantha polysaccharide. Int J Biol Macromol. 2020;156:1202-1216. doi:10.1016/j.ijbiomac.2019.11.158

16. Xiangfeng C, Jing D, Yongliang Z, et al. Comprehensive analysis of IncRNA and mRNA expression profiles in macrophages activated by actinidia eriantha polysaccharide. Int $J$ Biol Macromol. 2019;136:980-993. doi:10.1016/j.ijbiomac.2019.06.091

17. Postow MA, Callahan MK, Wolchok JD. Immune checkpoint blockade in cancer therapy. J Clin Oncol. 2015;33(17):1974-1982. doi:10.1200/JCO.2014.59.4358

18. Chinai JM, Janakiram M, Chen F, et al. New immunotherapies targeting the PD-1 pathway trends. Pharmacol Sci. 2015;36 (9):587-595. doi:10.1016/j.tips.2015.06.005
OncoTargets and Therapy

\section{Publish your work in this journal}

OncoTargets and Therapy is an international, peer-reviewed, open access journal focusing on the pathological basis of all cancers, potential targets for therapy and treatment protocols employed to improve the management of cancer patients. The journal also focuses on the impact of management programs and new therapeutic agents and protocols on patient perspectives such as quality of life, adherence and satisfaction. The manuscript management system is completely online and includes a very quick and fair peer-review system, which is all easy to use. Visit http://www.dovepress.com/ testimonials.php to read real quotes from published authors. 